\title{
The effects of reordering the response categories on the area under the ROC
}

\author{
LARRY KERR \\ Pennsylvania State University, University Park, Pennsylvania 16802 \\ and \\ JAMES P. THOMAS \\ University of California, Los Angeles, California 90024
}

\begin{abstract}
The area under the rating ROC can be a useful index of stimulus discriminability. It has the advantage that few assumptions must be made about the underlying distributions of signal and noise. One of the assumptions that must be made is the order of the subject's response scale. A procedure is outlined for determining the order of the response scale actually used by the subject, and some of the implications of reordering the scale used in analysis are discussed. Results of computer simulation of the effects of varying some important experimental parameters are presented.
\end{abstract}

The use of the area under the receiver operating characteristic curve (ROC) as a psychophysical measure of stimulus detectability or discriminability has a number of important advantages. One of the advantages is that the area under the ROC is numerically equivalent to percent correct in a two-alternative forced-choice procedure that is free from response bias (Green \& Swets, 1966); this property allows a simple, straightforward interpretation of results and ties them to data obtained with other methods. Another very useful property is that none of the assumptions regarding the underlying noise and signal-plus-noise distributions required by the d' measure are necessary; that is, the area under the ROC can be used as an empirical nonparametric means of comparing two distributions of responses, one distribution obtained on noise trials and the other on signal-plus-noise trials. This property is obviously useful when the nature of the response distribution is unknown. It is also useful when the object of the experiment is to assess the degree of discriminability of two types of stimuli (Bagrash, Thomas, \& Shimamura, 1974); in this case, one stimulus is arbitrarily designated as "signal" and the other as "noise," and the area under the ROC becomes a useful objective index of the subject's ability to discriminate between the stimuli. The use of the area under the ROC in conjunction with a rating method to measure stimulus discriminability is particularly powerful and efficient, since it allows pairwise comparisons to be derived from ranking or rating data generated from experimental sessions in which more than two stimuli are presented. The purpose of this paper is to discuss some applications of this procedure and some of the difficulties that may arise.

A number of recent studies (Hafter, Bourbon, Blocker, \& Tucker, 1969; Hafter, Carrier, \& Stephan,
1973; Kerr, 1974; Thomas \& Shimamura, 1974) have used variations of the rating method or the method of successive categories (see Schönemann \& Tucker, 1967; Torgerson, 1958) to generate discriminability data and then analyzed those data using an area under the ROC measure. Hafter and his colleagues asked subjects to estimate by means of an analog rating device the lateralization of auditory signals masked by noise under a number of diotic and dichotic listening conditions. The lateralization ratings were used to generate a "lateralization ROC," and the area under that ROC provided an index to the extent to which the signals could be discriminated.

Thomas and Shimamura (1974) presented subjects with a series of five just-detectable disks of light that varied in size, and asked their subjects to rate on a 6-point scale the apparent size of the stimuli; the object of the experiment was to determine whether threshold stimuli are discriminable on the basis of size. In the analysis of the rating data, one stimulus was arbitrarily designated as signal and another as noise, and the size ratings were used to construct a rating $R O C$; the area under the ROC was used as an indication of the degree to which the pattern of size judgments for the two stimuli differed. The analysis was repeated for all pairs of stimuli, yielding an index of discriminability for each pair. An important feature of this experiment is that, while all five stimuli were presented in the same experimental session (in random order), the analysis enabled the quantitative comparison of all possible pairs of stimuli in terms of their discriminability.

Kerr (1974) used essentially the same procedure with wavelength as the stimulus variable and had his subjects try to identify the wavelength of just-detectable stimuli. The pattern of responses for each stimulus was compared with the pattern of responses for each 
other stimulus by treating one member of a pair of stimuli as signal and the other as noise, and treating the responses as wavelength ratings to construct an ROC. The area under the ROC was taken as an index of the discriminability of the two stimuli being compared. Again, the efficiency of the method is evident: Five wavelengths were presented in random order within a session, but the analysis permitted a quantitative assessment of the discriminability of each stimulus from every other stimulus.

As stated above, the use of the area under the ROC measure does not require any of the usual assumptions of signal detection theory regarding the underlying distributions of noise and signal plus noise. It does, however, require that the subject's response scale be at least ordinal; that is, a higher rating for purposes of constructing the ROC must be used as higher by the subject. Under most circumstances, one can assume an ordinal response scale and instruct the subjects accordingly. In some cases, however, there is no meaningful a priori order in the data. For example, in the Hafter et al. (1969) study, the response device imposed a left-right ordering on the responses, but there was no reason to consider a left response to be higher or lower than a right response. The question was the extent to which a stimulus was lateralized, but the side was irrelevant. On a left-right scale, a stimulus that was not lateralized at all would produce a response in the middle. Figure 2 in the Hafter et al. (1969) study shows that stimuli that were lateralized yielded a bimodal distribution of responses on the left-right scale.

In other cases, there is little a priori reason to select one response scale over another. For example, in the Kerr (1974) wavelength discrimination study, it is not at all certain that the subjective scale of hue was in the same order as the physical scale of wavelength. Two possible subjective dimensions of hue are illustrated in Figure 1. In $A$, the subjective scale of hue is represented by a color circle, with the spectral hues arranged around its circumference. In $B$, the subjective scale of hue is ordered on a single continuum and is monotonically related to the physical spectrum. Consider the case of a subject attempting to discriminate among near-threshold hues of red, green, yellow, and blue. If the subjective scale is represented by a color circle, it would be expected that the blue stimulus would be confused with the red stimulus more often than with the yellow stimulus, as illustrated in the "unrolled" color circle in Figure IC; the ROC analysis illustrated in Figure 1C would indicate good discrimination between the blue and yellow stimuli. However, if the ordering of the response scale were treated as if it were monotonic with the physical spectrum, the "blue" response would be bimodally distributed, and, if the blue stimulus were designated as noise, then for a criterion indicated by the vertical dashed line in Figure 1D, all of the "blue" responses to the red stimulus would be treated as false alarms, leading to a lower estimate of discriminability than in the former case. The problem in this instance is that the order of the categories used by the subject in generating the data is not the same as the order used by the experimenter in analyzing them. Similarly, in the case of size judgments (Thomas \& Shimamura, 1974), there are subtle theoretical reasons why one might not wish to assume that the subjective dimension of the size of just-detectable stimuli is ordinally related to the objective dimension of size. There are any number of potential situations in which the area under the ROC might be a useful performance index, but in which the experimenter may be unwilling to assume that the subjective scale has the known order required for this analysis.

The information required to determine the category order used by the subject is contained in the data and can be retrieved in a simple manner. The order of the responses used by the subject is that order in which the relation between the response, $r_{i}$, and the relative number of times that response was used on signal as compared with noise trials, $p\left(r_{i} \mid s\right) / p\left(r_{i} \mid n\right)$, is monotonically increasing. ${ }^{1}$ The fraction $p\left(r_{i} \mid s\right) / p\left(r_{i} \mid n\right)$ is similar to a likelihood ratio, $P\left(r_{i} \mid s\right) / P\left(r_{i} \mid n\right)$. Before constructing the ROC, the response categories can be rearranged so that the relationship between the categories and the likelihood ratio is monotonically increasing. This procedure assures that the category rankings used in the analysis are the same as the subject used in producing the data. In practice, it is simpler to use the ratio $p\left(r_{i} \mid s\right) / p\left(r_{i}\right)=p\left(r_{i} \mid s\right) /\left[p\left(r_{i} \mid s\right)+p\left(r_{i} \mid n\right)\right]$, since this ratio varies between 0 and 1 , while the former ratio varies between 0 and $\infty$. Moreover, the latter ratio may be thought of as reflecting a subjective estimate of the a posteriori probability of a signal trial associated with each response category.
A

C

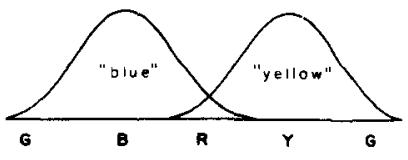

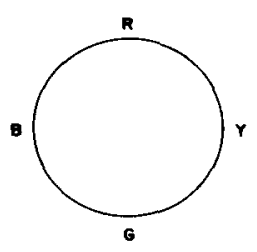

B

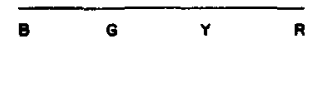

D

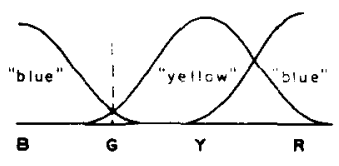

Figure 1. Illustration of two possible orders of a subjective scale of hue and the effect of imposing an incorrect ordering of response categories. (A) A color circle with the primary hues arranged about the circumference. (B) Spectral ordering of hues. (C) Distribution of responses "blue" and "yellow" on an unrolled color circle with blue and red adjacent. (D) Same distribution of responses but with a spectral ordering in which blue and red are at opposite ends of the scale. 
The process of reordering the response scale has the effect of maximizing the area under the ROC. ${ }^{2}$ If the original order is, in fact, the one that is used by the subject, the process of reordering has the undesirable effect of taking chance fluctuations of response proportions in each category and producing spuriously larger areas under the ROC. In the course of several visual detection experiments using a six-category confidence rating scale, the present authors have had the opportunity to examine the effects of reordering the response categories according to the way the subject used them, as compared to using the original experimenter-assigned ordering. Figure 2 shows the effect of reordering on the areas under the ROC for one subject. Solid symbols represent cases in which a small disk stimulus served as signal, and the open symbols represent cases in which no stimulus information was delivered, even on signal trials, that is, the designation of signal and noise was arbitrary. Reordering did not have much effect on the detection data for the small disk stimuli. In several cases, the order in which the subject used the ratings was the same as that assigned by the experimenter. In other cases, order reversals were found that had little effect on the ROC area when the stimuli being used were at or above the classically defined threshold, that is, when the area under the ROC was about midway between chance (.50) and perfect performance (1.0), or about .75. However, as the subject's performance became poorer, the effect of reordering became progressively

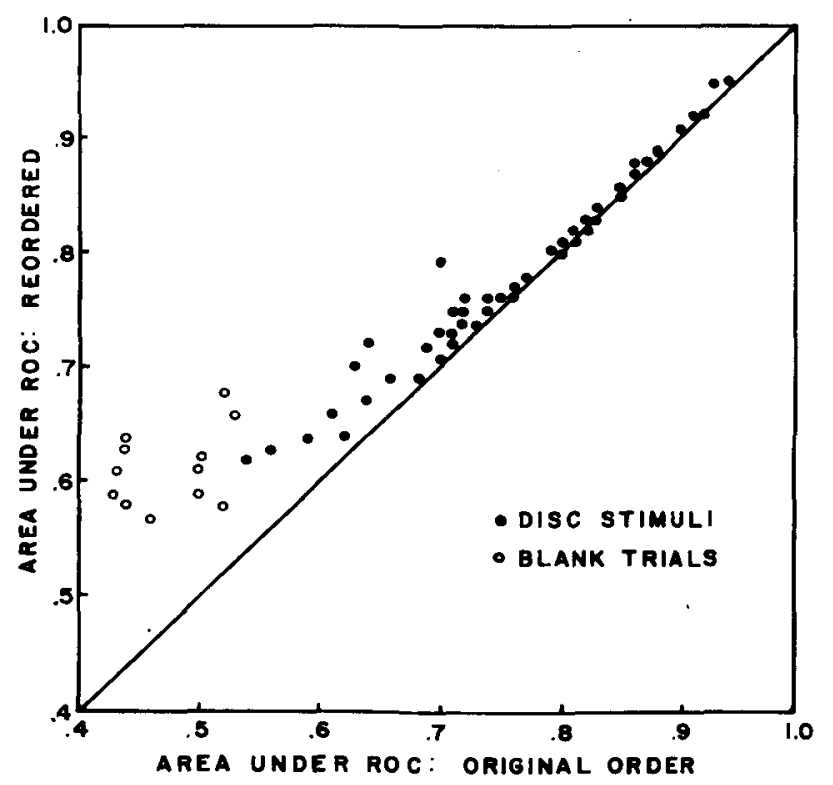

Figure 2. Comparison of areas under the ROC for reordered categories and original ordering. Solid symbols represent the case in which small disk stimuli were presented on signal trials; open symbols represent the case in which half of the blank trials were arbitrarily designated as signal trials. greater, reaching a maximum at chance performance levels. It is easy to see why this should be so. If the subject cannot distinguish between signal and noise trials, all deviations from equal frequency of use of any category on signal trials as compared to noise trials is due to sampling error. Since the process of reordering maximizes the area under the ROC, the sampling errors cannot cancel one another over categories. Instead, their effects are cumulated. Thus, where the originally ordered ROC would yield an area of .50 on the average for chance responding, the area under the reordered ROC can never be less than .50 and can only equal .50 when every category is used just as often on signal as on noise trials. As the subject's performance becomes poorer, the sampling errors have ever-increasing effects in determining the area under the reordered ROC.

The rating $R O C$ plots the cumulative proportions of responses in each category to signal and to noise trials on the two axes. In the case of chance performance (i.e., of random assignment of each response to either signal or to noise trials, as represented by the open symbols in Figure 2), the proportion of responses in each category to either signal or noise trials is binomially distributed, with the expected proportion $\mathrm{p}_{i}$ indicating the subject's response bias for that category. The variance of the proportion is $p_{i} q_{i} / n_{s}$ or $p_{i} q_{i} / n_{n}$, where $n_{s}$ and $n_{n}$ are the total number of signal or noise trials, respectively. Since, in the case of random responding, $p_{i}$ is the same for signal and for noise trials, the expectation is that, within any category, the proportion of responses will be the same for signal as for noise trials. The variance of the proportions on signal and noise trials depends on the relative number of each type of trial, as well, and will not be equal for different numbers of signal and noise trials. Thus, the factors that influence the variability of the response proportions in each category on both kinds of trials, and, therefore, influence the area under the reordered ROC, are the total number of trials, the relative number of signal and noise trials, and the subject's response bias.

Increasing the total number of trials decreases the variance of each sample proportion. Changing the relative number of signal and noise trials has the effect of increasing the number of one type of trial, while decreasing the number of the other type of trial, thereby increasing the variability of the proportion of responses in each category to one type of trial and decreasing the variability in the other type. Since the effect of changing the number of trials is not symmetrical, that is, increasing $\mathrm{n}$ has less relative effect on $\mathrm{pq} / \mathrm{n}$ than does decreasing $n$, the net effect of altering the relative number of signal and noise trials from equality is to increase the variability of the corresponding point on the ROC. ${ }^{3}$ The subject's bias will affect the variability by changing the value of $\mathrm{pq}$; as the 
probability of a response in any category changes from .5 , the variance will decrease. For a given decrease in response probability in one category, there must be a corresponding increase in the probability of a response in at least one other category, but again the effect on the variance is asymmetric, in that decreasing $p$ from some value smaller than .5 has a greater effect on the variance than does increasing $p$ from the same value. Thus, the greatest total variability in all response categories will occur when responses in all categories are equally likely.

The precise effects of changing these parameters on the area under the reordered ROC for the case of random responding are complex and difficult to predict. However, the present authors were able to simulate on a computer the behavior of a subject in such an experiment and to compute the areas under the reordered ROC for the worst case (i.e., a subject having no information regarding signal and noise trials and assigning responses to the categories in a random manner). ${ }^{4}$ The effects of changing the number of trials, the relative proportions of signal and noise trials, and the subject's response bias could thus be evaluated.

Some results are shown in Table 1. Each entry in the body of the table is the mean area under the reordered ROC for 100 simulated experiments in which the subject responded to each trial in a random manner with a certain probability $\mathrm{P}_{\mathbf{i}}$, in each of six categories. If the reordering had not been performed, these entries would all be within sampling error of .50 . The number of trials in each experiment is indicated in the left-hand column in the table. The total number of trials in each simulated experiment were either evenly divided between signal and noise trials or were divided as $75 \%$ signal and $25 \%$ noise, as indicated at the top of the table. Finally, two different kinds of response bias were simulated, one with approximately equal probability of response in each category $\left(P_{i}=.17\right.$ in Categories $1-4$ and $P_{i}=.16$ in Categories 5 and 6), and one whose distribution was severely skewed toward higher categories $\left(P_{i}\right.$ in Categories 1.6 was $.50, .30, .10$, $.05, .05$, and 0 ). The skewing had the effect of simul-

Table 1

Effect of Reordering the Response Categories on Area Under the ROC for Computer-Stimulated Random Responding

Signal-Noise Distribution

\begin{tabular}{rccccc}
\cline { 5 - 5 } $\begin{array}{c}\text { Number of } \\
\text { Trials }\end{array}$ & \multicolumn{2}{c}{$50 \%-50 \%$} & & \multicolumn{2}{c}{$75 \%-25 \%$} \\
\cline { 2 - 3 } \cline { 5 - 5 } & Uniform & Skewed & & Uniform & Skewed \\
\hline 30 & .7091 & .6494 & & .7380 & .6728 \\
50 & .6688 & .6219 & & .6926 & .6472 \\
100 & .6183 & .5866 & & .6399 & .6018 \\
200 & .5821 & .5593 & & .5953 & .5709 \\
500 & .5505 & .5370 & & .5610 & .5450 \\
1,000 & .5351 & .5275 & .5415 & .5308 \\
\hline
\end{tabular}

Table 2

Effects of Reordering the Response Categories on Area Under the ROC for Actual Subjects' Responses to Blank Trials Arbitrarily Designated "Signal" and "Noise"

\begin{tabular}{cccc}
\hline Subject & $\begin{array}{c}\text { Number of } \\
\text { Samples }\end{array}$ & $\begin{array}{c}\text { Number of } \\
\text { Trials }\end{array}$ & $\begin{array}{c}\text { Mean Area } \\
\text { Under ROC }\end{array}$ \\
\hline BT & 146 & 80 & .6244 \\
J H & 146 & 80 & .5946 \\
J T & 142 & 80 & .6201 \\
J T & 110 & 100 & .6008 \\
KS & 139 & 100 & .6164 \\
\hline
\end{tabular}

taneously reducing the number of response categories to five and introducing a severe response bias. The effect of changing the number of categories was also simulated directly, although these data are not included in the table. Other factors being equal, increasing the number of categories monotonically increased the area under the reordered ROC, at least up to six categories.

As shown in Table 1, the expected effects of changing the above parameters are obtained in the simulation. Increasing the number of trials reduces the effect of reordering for all conditions; changing the relative number of signal and noise trials from equality increases the effect of reordering; and introducing a response bias decreases the reordering effect. In an experiment, the experimenter has direct control over the number of trials and the relative number of signal and noise trials. To minimize the effect of random errors in reordering the response categories, a large number of trials should be used with an equal number of signal and noise trials whenever possible. In addition, the subject can be urged to try to distribute his responses evenly across categories.

It is of interest to see how the computer simulation results compare with data obtained from real subjects in an experiment. While the present authors have not tried an experiment in which only blank trials were presented, the noise trials in a detection experiment have been arbitrarily divided into two groups and the responses to these trials analyzed in a manner similar to that used in the computer simulation. That is, half of the blank trials were designated signal and the other half noise, and the area under the reordered ROC was computed. The results are presented in Table 2. The column headed "Number of Samples" shows the total number of separate experimental sessions on which the analysis was performed for each subject, with the number of trials in each of the sessions shown in the next column. The trials were always evenly divided between signal and noise trials; thus, 80 trials means 40 signal and 40 noise trials. Since the trials were presented during a series of actual stimulus presentations, the subjects did not respond to the blank trials equally often in each category, but rather they used the lower categories much more frequently, introducing a severe response bias into the data. 
Comparison of these data with those presented in Table 1 shows general agreement for comparable numbers of trials, although considering the skewed response distribution in the real subject data, those areas seem slightly larger than was expected.

The application of the foregoing analysis to discriminability data is direct and straightforward. In fact, the data of Kerr (1974) and Thomas and Shimamura (1974) were subjected to category reordering with the result that reordering had little or no effect on the discriminability measure. This finding indicates that the original category order along the physical dimension was the category order that the subjects in fact used in the experiments. However, with the process of category reordering, such a correspondence was not essential to the use of the area under the ROC measure as an index of stimulus discriminability.

\section{REFERENCES}

Bagrash, F. M., Thomas, J. P., \& Shimamura, K. K. Sizetuned mechanisms: Correlation of data on detection and apparent size. Vision Research, 1974, 14, 937-942.

GREEN, D. M., \& SwETs, J. A. Signal detection theory and psychophysics. New York: Wiley, 1966.

Hafter, E. R., Bourbon, W. T., Blocker, A. S., \& TUCKER, A. A direct comparison between lateralization and detection under conditions of antiphasic masking. Journal of Acoustical Society of America, 1969, 46, $1452-1457$.

Hafter, E. R., Carrier, S. C., \& Stephan, F. K. Direct comparison of lateralization and the MLD for monaural signals in gated noise. Journal of Acoustical Society of America, 1973, 53, 1553-1559.

KERR, L. G. Detection and identification of monochromatic stimuli under chromatic contrast. Vision Research, 1974, 14, 1095-1105.

Thomas, J. P., \& Shimamura, K. K. Perception of size at the detection threshold: Its accuracy and possible mechanisms. Vision Research, 1974, 14, 535-543.

TORGERson, W. S. Theory and methods of scaling. New York: Wiley, 1958.

SchönemanN, P. H., \& Tucker, L. R. A maximum likelihood solution for the method of successive intervals allowing for unequal stimulus dispersions. Psychometrika, 1967, 32, 403-417.

\section{NOTES}

1. The higher a category, the more likely that category is to be used on signal trials as compared with noise trials; the lower the category, the less likely it is to be used on signal trials as compared with noise trials. Thus, if the order used by the subject is, for example, $a, b, c, d, e, f$, then,

$$
\frac{p\left(r_{d} \mid s\right)}{p\left(r_{d} \mid n\right)} \geqslant \frac{p\left(r_{c} \mid s\right)}{p\left(r_{c} \mid n\right)}
$$

but, if the order used by the subject is, for example, f, e, d, c, b, a, then,
The order of categories required to arrange the fraction $p\left(r_{i} \mid s\right) / p\left(r_{i} \mid n\right)$ in monotonically increasing order is the order of categories used by the subject.

2 . The rating $R O C$ plots on the abscissa the cumulative proportion of responses in each category or a higher ordered category on noise trials and plots on the ordinate the corresponding cumulative proportion on signal trials. In other words, the coordinates for one point on a 6-point rating ROC are the proportions of responses in Category 6 on noise and signal trials, respectively; the coordinates for the next point are the proportions of responses in Categories 5 and 6 on noise and signal trials; the coordinates for the next point are the proportions of responses in Categories 4,5 , and 6 on noise and signal trials, and so on. When the subject uses the experimenter-assigned order, such a procedure results in the familiar negatively accelerating ROC. The area under the ROC will be maximum when the increase on the ordinate relative to the increase on the abscissa is greatest for the higher categories, that is, at the lower left of the ROC, and smallest for the lower categories, that is, at the upper right. This will occur for that order of categories, $a, b, c, d, e, f$, in which

$$
\frac{p\left(r_{f} \mid s\right)}{p\left(r_{f} \mid n\right)} \geqslant \frac{p\left(r_{e} \mid s\right)+p\left(r_{f} \mid s\right)}{p\left(r_{e} \mid n\right)+p\left(r_{f} \mid n\right)} \geqslant \frac{p\left(r_{d} \mid s\right)+p\left(r_{e} \mid s\right)+p\left(r_{f} \mid s\right)}{p\left(r_{d} \mid n\right)+p\left(r_{e} \mid n\right)+p\left(r_{f} \mid n\right)},
$$

and so on. That this category order is the same as that produced by reordering according to the above criterion can be shown as follows:

$$
\frac{p\left(r_{f} \mid s\right)}{p\left(r_{f} \mid n\right)} \geqslant \frac{p\left(r_{e} \mid s\right)+p\left(r_{f} \mid s\right)}{p\left(r_{e} \mid n\right)+p\left(r_{f} \mid n\right)} .
$$

By cross-multiplying,

$$
\begin{gathered}
{\left[p\left(r_{f} \mid s\right) \cdot p\left(r_{e} \mid n\right)\right]+\left[p\left(r_{f} \mid s\right) \cdot p\left(r_{f} \mid n\right)\right] \geqslant} \\
{\left[p\left(r_{e} \mid s\right) \cdot p\left(r_{f} \mid n\right)\right]+\left[p\left(r_{f} \mid s\right) \cdot p\left(r_{f} \mid n\right)\right]} \\
p\left(r_{f} \mid s\right) \cdot p\left(r_{e} \mid n\right) \geqslant p\left(r_{e} \mid s\right) \cdot p\left(r_{f} \mid n\right) \\
\frac{p\left(r_{f} \mid s\right)}{p\left(r_{f} \mid n\right)} \geqslant \frac{p\left(r_{e} \mid s\right)}{p\left(r_{e} \mid n\right)},
\end{gathered}
$$

which is the criterion for reordering. A similar proof can be constructed for the other categories.

3. This follows from the observation that

$$
\left|\frac{1}{n}-\frac{1}{n+k}\right|<\left|\frac{1}{n}-\frac{1}{n-k}\right| .
$$

The decrease in variance on one axis of the ROC that results from an increase in $\mathbf{n}$ on that axis is less than the increase in variance on the other axis that results from the decrease in $n$ on that axis, and the total variability will be least when $n_{s}=n_{n}$.

4. By "worst case" is meant the situation in which the effects of reordering will have the greatest effect on cumulating random fluctuations in response categories and spuriously increasing the area under the ROC. These effects will obviously be maximum when the random component in the response proportions in each category is maximum, that is, when the subject is responding randomly.

$$
\frac{p\left(r_{d} \mid s\right)}{p\left(r_{d} \mid n\right)} \leqslant \frac{p\left(r_{c} \mid s\right)}{p\left(r_{c} \mid n\right)}
$$

